\title{
Control of Nonlinear System - Adaptive and Predictive Control
}

\author{
Jiri Vojtesek*, Petr Dostal*, Vladimir Bobal* \\ *Department of Process Control, Faculty of Applied Informatics, \\ Tomas Bata University in Zlin, Czech Republic \\ (Tel: 00420576035199; e-mail: \{vojtesek,dostalp,bobal\}@fai.utb.cz)
}

\begin{abstract}
The goal of this paper is to propose suitable control methods for controlling of the highly nonlinear system represented by the mathematical model of the continuous stirred tank reactor (CSTR) with so called van der Vusse reaction inside. Temperature of the reactant is controlled by the heat removal of the cooling liquid in the reactor's jacket. Two control strategies were suggested - adaptive control and predictive control. The adaptive approach uses recursive identification for the optimal setting of the controller. The predictive control computes input sequence by the minimizing of the cost function constructed by the difference between output variable and reference signal. Both control strategies shows good control results and pertinence for the controlling of such type of systems.
\end{abstract}

Keywords: Adaptive control, Predictive Control, Polynomial methods, Recursive estimation, Nonlinear systems, CSTR, Simulation.

\section{INTRODUCTION}

Unfortunately, most of the processes in the technical praxis have nonlinear properties. Typical example of the nonlinear system can be found in the chemical or the biochemical industry where so called chemical reactor is used for production of the several chemicals or drugs (Corriou, 2004).

Controlling of these devices with the conventional methods where parameters of the controller are set at the beginning fixed during the control could result in non-optimal control responses because of changing parameters of the system. This inconvenience could be overcome with use of other control strategies which takes into account these changes, for example adaptive or predictive control. These two control strategies are compared in this paper in order to compare obtained simulation results.

The basic idea of adaptive control is that parameters or the structure of the controller are adapted to parameters of the controlled plant according to the selected criterion (Bobál et al., 2005). Adaptation can be done for example by the modification of the controller's parameters by the change of the controller's structure or by generating an appropriate input signal, which is called "adaptation by the input signal".

The polynomial synthesis (Kučera, 1993) is one of the methods used in adaptive control for control synthesis of the system. This method is based on the input-output model of the controlled system or its transfer function. It can be classified as an algebraic method and is based on algebraic operations in the ring of polynomials. Polynomials are usually described in $s$-plane for continuous systems, in $z$ plane for discrete systems and in $\delta$-plane for systems which come from $\delta$-models of both the controlled system and the controller too (Middleton and Goodwin, 2004) and (Mukhopadhyay et al., 1992).
One of the biggest advantages of the polynomial method compared to the conventional method is that it provides not only relations for computing of the controller's parameters but the structure of the controller too. This structure fulfils general requirements for control systems and input signals (reference signal and disturbance) and it can be used for controlling of the systems with negative properties from the control point of view, such as non-minimum phase systems or unstable systems. Another advantage is that the resulted relations are easily programmable.

Polynomials in the numerator and denominator of the transfer function of the controller result from the solution of Diophantine equations, which have so called characteristic polynomial of the closed loop system on the right side of the equation. The roots of this polynomial are then poles of the closed-loop system, which affects the quality of control. The method of choosing the poles is called Pole-placement or Pole-assignment (Kučera, 1991).

The idea of the predictive control is based on the calculation of the control sequence from the actual time point minimizing the deviation of the reference signal and the output signal of the plant in the future horizon (Clarke et al., 1987). The future values of the reference signal are given in advance or are assumed to be equal to the present one. The future values of the plant can be predicted from a process model. If disturbances are measurable, then their future values are predicted using some assumptions.

All approaches are verified by the simulation in the simulation program Matlab®, version 6.5.

\section{ADAPTIVE CONTROL}

The adaptive approach in this work is based on choosing an external linear model (ELM) of the original nonlinear system 
whose parameters are recursively identified during the control. Parameters of the resulted continuous controller are recomputed in every step from the estimated parameters of the ELM (Bobál et al., 2005).

\subsection{External Linear Model}

The main types of ELM are continuous-time (CT) models and discrete-time (DT) models.

The general description of the CT ELM can be formulated via transfer function $G(s)$ :

$$
G(s)=\frac{b(s)}{a(s)}=\frac{Y(s)}{U(s)}=\frac{b_{m} s^{m}+b_{m-1} s^{m-1}+\ldots+b_{1} s+b_{0}}{a_{n} s^{n}+a_{n-1} s^{n-1}+\ldots+a_{1} s+a_{0}}
$$

The continuous-time ELM is supposed to be more accurate and corresponding to the real model because data are estimated continuously during the control. On the contrary, $\mathrm{CT}$ identification is difficult.

On the other hand, identification of the DT models are easy to realize. We can say that discrete models are used in the cases where the usage of continuous ones is complicated or the realization is impossible. An important variable in the discrete-time models is sampling period $T_{v}$.

The transfer function $G$ in this case is defined as $Z$-transform of the output variable $y$ to the input variable $u$

$$
G(z)=\frac{Y(z)}{U(z)}=\frac{b(z)}{a(z)}=\frac{b_{m} z^{m}+b_{m-1} z^{m-1}+\ldots+b_{1} z+b_{0}}{a_{n} z^{n}+a_{n-1} z^{n-1}+\ldots+a_{1} z+a_{0}}
$$

where $a(z)$ and $b(z)$ are discrete polynomials and $U(z)$ and $Y(z)$ are $Z$-transform images of the input and output variables.

\subsection{Identification}

The use of the discrete model for nonlinear system can cause problems with the sampling period $T_{v}$. This sampling period cannot be small because of the stability and the big sampling period is unacceptable because we do not know what will happen with the system during this sample.

The inconvenience with the sampling period could be overcome with the use of so called delta $(\delta-)$ models. Although the delta operator belongs to the class of discrete models with the operator described as

$q \cdot x(k) \triangleq x(k+1)$

it can been seen from

$\delta=\frac{q-1}{T_{v}}$

that this operator is related to sampling period $T_{v}$ and it means that $\delta$-models are close to the continuous ones in $d / d t$.

A new complex variable in " $\delta$ " plane called " $\gamma$ ", which is defined for example in (Mukhopadhyay et al., 1992) as $\gamma=\frac{z-1}{\beta \cdot T_{v} \cdot z+(1-\beta) \cdot T_{v}}$

need to be introduced. We can obtain an infinite number of $\delta$-models for different values of optional parameter $\beta$ in Equation (5) from the range $0 \leq \beta \leq 1$.

The forward $\delta$-model described for $\beta=0$ by

$\gamma=\frac{z-1}{T_{v}}$

is dealt with in this work.

The Recursive Least-Squares (RLS) method is used for the parameter estimation in this work. The RLS method is wellknown and widely used for the parameter estimation (Fikar and Mikleš, 1999). It is usually modified with some kind of forgetting, exponential or directional (Kulhavý and Karny, 1984), because parameters of the identified system can vary during the control which is typical for nonlinear systems and the use of some forgetting factor could result in better output response.

The RLS method with exponential forgetting is describe by the set of equations:

$$
\begin{aligned}
& \varepsilon(k)=y(k)-\varphi^{T}(k) \cdot \hat{\theta}(k-1) \\
& \gamma(k)=\left[1+\varphi^{T}(k) \cdot \mathbf{P}(k-1) \cdot \varphi(k)\right]^{-1} \\
& \mathrm{~L}(k)=\gamma(k) \cdot \mathbf{P}(k-1) \cdot \varphi(k) \\
& \mathbf{P}(k)=\frac{1}{\lambda_{1}(k-1)}\left[\mathbf{P}(k-1)-\frac{\mathbf{P}(k-1) \cdot \varphi(k) \cdot \varphi^{T}(k) \cdot \mathbf{P}(k-1)}{\lambda_{1}(k-1)+\varphi^{T}(k) \cdot \mathbf{P}(k-1) \cdot \varphi(k)}\right] \\
& \hat{\theta}(k)=\hat{\theta}(k-1)+\mathrm{L}(k) \varepsilon(k)
\end{aligned}
$$

Several types of exponential forgetting can be used, e.g. like RLS with constant exponential forgetting, RLS with increasing exp. forgetting etc. RLS with the changing exp. forgetting is used for parameter estimation, where the changing forgetting factor $\lambda_{1}$ is computed from the equation

$\lambda_{1}(k)=1-K \cdot \gamma(k) \cdot \varepsilon^{2}(k)$

Where $K$ is small number, in our case $K=0.001$.

\subsection{Polynomial Synthesis}

(3) The structure of the controller is designed via polynomial synthesis. The simple one degree-of-freedom (1DOF) control configuration was used. The block scheme of this configuration in Fig. 1.

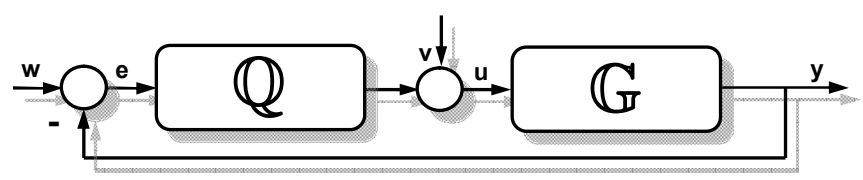

Fig. 1 1DOF control configuration

Block $Q$ in Fig. 1 represents the transfer function of the controller, $G$ denotes the transfer function of the plant, $w$ is 
the reference signal, $e$ is used for the control error, $v$ is the disturbance at the input to the system, $u$ determines the input variable, and finally $y$ is the output variable.

Transfer functions of the controller and controlled plant could be described in the continuous time by equations:

$$
Q(s)=\frac{q(s)}{s \cdot p(s)} ; G(s)=\frac{b(s)}{a(s)}
$$

where polynomials $p(s)$ and $q(s)$ are designed by the polynomial approach and parameters of these polynomials are computed by the Method of uncertain coefficients which compares coefficients of individual $s$-powers from Diophantine equation (Kučera, 1993):

$$
a(s) \cdot s \cdot p(s)+b(s) \cdot q(s)=d(s)
$$

Although parameters of the polynomials $a(s)$ and $b(s)$ are reflected to be in continuous-time, the identification runs recursively in discrete time periods related to the sampling period $T_{v}$. This simplification is supported by the use of $\delta$ models where each input and output variable is recomputed to this sampling period, $T_{v}$, which shifts these discrete polynomials closer to the continuous ones. It was proofed for example in (Stericker and Sinha, 1993) that the parameters of the delta model for the small sampling period approach to the continuous ones in (9).

The feedback controller $Q(s)$ in Fig. 1 ensures all basic control requirements - i.e. stability, load disturbance attenuation and asymptotic tracking of the reference signal. It is required that each controller could be tuned somehow. This option can be found in this controller in the stable optional polynomial $d(s)$ on the right side of the Diophantine equation (10). As it is mentioned above, there are several methods for choosing of this polynomial. The method used here is Poleplacement or Pole-assignment method. Polynomial $d(s)$ can be divided into two parts $-m(s)$ and $n(s)$, so

$$
d(s)=m(s) \cdot n(s)
$$

where polynomial $n(s)$ is computed from the spectral factorization of polynomial $a(s)$ in the denominator of the transfer function $G(s)(9)$

$$
n^{*}(s) \cdot n(s)=a^{*}(s) \cdot a(s)
$$

and polynomial $m(s)$ is a stable one $m(s)=\left(s+\alpha_{i}\right)^{\operatorname{deg} d-\operatorname{deg} n}$ and $\alpha_{i}>0$ are $(\operatorname{deg} d-\operatorname{deg} n)$ optional stable roots, usually called poles of the control system. A disadvantage of this method can be found in the uncertainty of the polynomial $m(s)$ - there is no general rule how to choose roots $\alpha_{l}$.

\section{PREDICTIVE CONTROL}

\subsection{Generalized Predictive Control}

Generalized Predictive Control (GPC) is one of the most popular predictive methods based on Model Predictive
Control (MPC) (Clarke et al., 1987), and has been successfully used in praxis for different types of control problems from this time.

The GPC has many common ideas with the ordinary predictive methods but it has some differences to such as the solution of the GPC controller is analytical, it can be used for unstable and non-minimum phase systems etc.

The general single-input single-output (SISO) after linearization can be described through the discrete backshift operators $z^{-1}$ as

$$
A\left(z^{-1}\right) \cdot y(t)=z^{-d} \cdot B\left(z^{-1}\right) \cdot u(t-1)+C\left(z^{-1}\right) \cdot e(t)
$$

where $u(t)$ is control variable, $y(t)$ output variable, $e(t)$ denotes a zero mean white noise, and $d$ is dead time of the system. Polynomials $A\left(z^{-1}\right), B\left(z^{-1}\right)$ and $C\left(z^{-1}\right)$ are

$$
\begin{aligned}
& A\left(z^{-1}\right)=1+a_{1} z^{-1}+a_{2} z^{-2}+\ldots+a_{n_{a}} z^{-n_{a}} \\
& B\left(z^{-1}\right)=b_{0}+b_{1} z^{-1}+b_{2} z^{-2}+\ldots+b_{n_{b}} z^{-n_{b}} \\
& C\left(z^{-1}\right)=1+c_{1} z^{-1}+c_{2} z^{-2}+\ldots+c_{n_{c}} z^{-n_{c}}
\end{aligned}
$$

Equation (23) is called the Controller Auto-Regressive Moving-Average (CARMA) model. This model is not suitable in most industrial processes where disturbances are non-stationary. In these cases, the integrated CARMA (CARIMA) model is more suitable

$$
A\left(z^{-1}\right) \cdot y(t)=z^{-d} \cdot B\left(z^{-1}\right) \cdot u(t-1)+C\left(z^{-1}\right) \cdot \frac{e(t)}{\Delta}
$$

where $\Delta=1-z^{-1}$.

The GPC algorithm can be then formulated as minimization of the cost function

$$
\begin{gathered}
J_{G P C}=\sum_{j=N_{1}}^{N_{2}} \delta_{u}(j)[\hat{y}(t+j \mid t)-w(t+j)]^{2}+\ldots \\
\ldots+\sum_{j=1}^{N_{u}} \lambda_{u}(j)[\Delta u(t+j-1)]^{2}
\end{gathered}
$$

where $\hat{y}(t+j \mid t)$ is an optimum $j$-ahead prediction of the output on data up to time $t$, further, $N_{1}$ and $N_{2}$ denote minimum and maximum costing horizons, respectively, $N_{u}$ is control horizon, $w(t+j)$ means reference signal, $\Delta u$ stands for manipulated variable and finally $\delta_{u}(j)$ and $\lambda_{u}(j)$ denote weighting sequences.

The values of these factors are for simplification assigned as $\delta_{u}=1$, and $\lambda_{u}$ is constant through the whole time interval of the control.

\section{MODEL OF THE PLANT}

The nonlinear system under the consideration is the Continuous Stirred Tank Reactor (CSTR). The reaction inside the reactor is called van der Vusse reaction can be described by the following reaction scheme (Chen, et al., 1995): 
$A \stackrel{k_{1}}{\longrightarrow} B \stackrel{k_{2}}{\longrightarrow} C$

$2 A \stackrel{k_{3}}{\longrightarrow} D$

(17)

The graphical scheme of this reactor can be seen in Fig. 2

The mathematical model of this reactor is described by the following set of ordinary differential equations (ODE):

$\frac{d c_{A}}{d t}=\frac{q_{r}}{V_{r}}\left(c_{A 0}-c_{A}\right)-k_{1} c_{A}-k_{3} c_{A}^{2}$

$\frac{d c_{B}}{d t}=-\frac{q_{r}}{V_{r}} c_{B}+k_{1} c_{A}-k_{2} c_{B}$

$\frac{d T_{r}}{d t}=\frac{q_{r}}{V_{r}}\left(T_{r 0}-T_{r}\right)-\frac{h_{r}}{\rho_{r} c_{p r}}+\frac{A_{r} U}{V_{r} \rho_{r} c_{p r}}\left(T_{c}-T_{r}\right)$

$\frac{d T_{c}}{d t}=\frac{1}{m_{c} c_{p c}}\left(Q_{c}+A_{r} U\left(T_{r}-T_{c}\right)\right)$

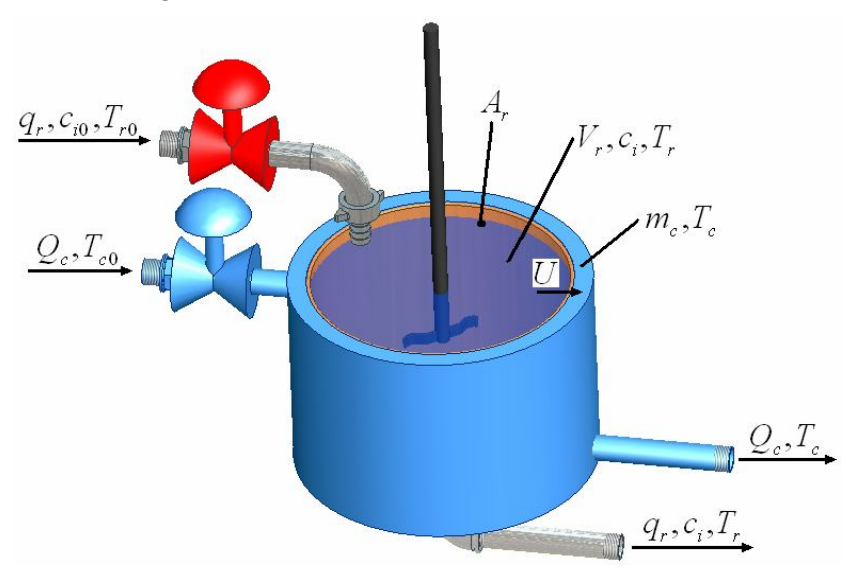

Fig. 2: Continuous Stirred Tank Reactor (CSTR)

This set of ODE together with simplifications then mathematically represents examined CSTR reactor. The model of the reactor belongs to the class of lumpedparameter nonlinear systems. Fixed parameters of the system are shown in Table 1 (Chen, et al., 1995).

Table 1 Fixed parameters of CSTR

\begin{tabular}{|c|c|}
\hline $\begin{array}{c}k_{01}=2.145 \cdot 10^{10} \mathrm{~min}^{-1} \\
k_{03}=1.5072 \cdot 10^{8} \mathrm{~min}^{-1} \cdot \mathrm{mol}^{-1} \\
E_{2} / R=9758.3 \mathrm{~K} \\
h_{1}=-4200 \mathrm{~kJ} . \mathrm{kmol}^{-1} \\
h_{3}=41850 \mathrm{~kJ} . \mathrm{kmol}^{-1} \\
V_{r}=0.01 \mathrm{~m}^{3} \\
c_{p r}=3.01 \mathrm{~kJ} \cdot \mathrm{kg}^{-1} \cdot \mathrm{K}^{-1} \\
c_{p c}=2.0 \mathrm{~kJ} . \mathrm{kg}^{-1} \cdot \mathrm{K}^{-1} \\
U=67.2 \mathrm{~kJ} . \mathrm{min}^{-1} \mathrm{~m}^{-2} \mathrm{~K}^{-1} \\
c_{A 0}=5.1 \mathrm{kmolm}^{-3} \\
T_{r 0}=387.05 \mathrm{~K}\end{array}$ & $\begin{array}{c}k_{02}=2.145 \cdot 10^{10} \mathrm{~min}^{-1} \\
E_{1} / R=9758.3 \mathrm{~K} \\
E_{3} / R=8560 \mathrm{~K} \\
h_{2}=11000 \mathrm{~kJ} . \mathrm{kmol}^{-1} \\
\\
\rho_{r}=934.2 \mathrm{~kg} . \mathrm{m}^{-3} \\
q_{r}=2.365 \cdot 10^{-3} \mathrm{~m}^{3} \mathrm{~min}^{-1} \\
Q_{c}=-18.5583 \mathrm{~kJ}_{\mathrm{min}} \mathrm{min}^{-1} \\
A_{r}=0.215 \mathrm{~m}^{2} \\
c_{B 0}=0 \mathrm{kmol} . \mathrm{m}^{-3} \\
m_{c}=5 \mathrm{~kg}\end{array}$ \\
\hline
\end{tabular}

The reaction heat $\left(h_{r}\right)$ in eq. (20) is expressed as:

$h_{r}=h_{1} \cdot k_{1} \cdot c_{A}+h_{2} \cdot k_{2} \cdot c_{B}+h_{3} \cdot k_{3} \cdot c_{A}^{2}$

where $h_{i}$ means reaction enthalpies.

Nonlinearity can be found in reaction rates $\left(k_{j}\right)$ which are described via Arrhenius law:
$k_{j}\left(T_{r}\right)=k_{0 j} \cdot \exp \left(\frac{-E_{j}}{R T_{r}}\right)$, for $j=1,2,3$

where $k_{0}$ represent pre-exponential factors and $E$ are activation energies.

Static analysis has shown (Vojtesek, et al., 2004), that system has an optimal working point for volumetric flow rate of the reactant $q_{r}=2.365 \times 10^{-3} \mathrm{~m}^{3} \cdot \mathrm{min}^{-1}$ a heat removal $Q_{c}=-18.56$ $k J \cdot \mathrm{min}^{-1}$. The difference between actual and initial temperature of the reactant $T_{r}$ was taken as controlled output and changes of the heat removal $Q_{c}$ was set as control input, i.e.

$$
\begin{aligned}
& y(t)=T_{r}(t)-T_{r}^{s}(t)[K] \\
& u(t)=100 \cdot \frac{Q_{c}(t)-Q_{c}^{s}(t)}{Q_{c}^{s}(t)}[\%]
\end{aligned}
$$

On the other hand, dynamic analysis results in ELM represented by a second order transfer function with relative order one, which is generally:

$G(s)=\frac{b(s)}{a(s)}=\frac{b_{1} s+b_{0}}{s^{2}+a_{1} s+a_{0}}$

Equation (25) can be rewritten for the identification to the form of the differential equation

$$
\begin{aligned}
y_{\delta}(k)= & -a_{1} y_{\delta}(k-1)-a_{0} y_{\delta}(k-2)+ \\
& +b_{1} u_{\delta}(k-1)+b_{0} u_{\delta}(k-2)
\end{aligned}
$$

where $y_{\delta}$ is recomputed output to the $\delta$-model:

$$
\begin{gathered}
y_{\delta}(k)=\frac{y(k)-2 y(k-1)+y(k-2)}{T_{v}^{2}} \\
y_{\delta}(k-1)=\frac{y(k-1)-y(k-2)}{T_{v}} \quad y_{\delta}(k-2)=y(k-2) \\
u_{\delta}(k-1)=\frac{u(k-1)-u(k-2)}{T_{v}} \quad u_{\delta}(k-2)=u(k-2)
\end{gathered}
$$

where $T_{v}$ is the sampling period, the data vector is

$\phi^{T}(k-1)=\left[-y_{\delta}(k-1),-y_{\delta}(k-2), u_{\delta}(k-1), u_{\delta}(k-2)\right]$

and the vector of estimated parameters

$\hat{\Theta}^{T}(k)=\left[\hat{a}_{1}, \hat{a}_{0}, \hat{b}_{1}, \hat{b}_{0}\right]$

could be computed from the ARX (Auto-Regressive eXogenous) model

$y_{\delta}(k)=\hat{\Theta}^{T}(k) \phi(k-1)$

by the recursive least squares methods described in part 2.2.

The ELM is of the second order, which means that degrees of polynomials $p(s), q(s)$, and $d(s)$ are then:

$\operatorname{deg} q=2 ; \operatorname{deg} p=1 ; \operatorname{deg} d=4$

and polynomials $m(s)$ and $n(s)$ in the equation (11) are

$n(s)=s^{2}+n_{1} s+n_{0} ; \quad m(s)=\left(s+\alpha_{i}\right)^{\operatorname{deg} d-\operatorname{deg} n}=\left(s+\alpha_{i}\right)^{2}$ 
and coefficients of the polynomial $n(s)$ are computed via spectral factorization (12) as

$$
n_{0}=\sqrt{a_{0}^{2}}, n_{1}=\sqrt{2 n_{0}+a_{1}^{2}-2 a_{0}}
$$

Transfer functions of the feedback and feedforward parts of the controller for $1 \mathrm{DOF}$ and $2 \mathrm{DOF}$ configurations are

$Q(s)=\frac{q_{2} s^{2}+q_{1} s+q_{0}}{s\left(s+p_{0}\right)}$

Where parameters of the polynomials $q(s)$ and $p(s)$ by the comparison of the coefficients of the $s$-powers a in diophantine equations (10).

The identified parameters of the delta ELM from the predictive control were recomputed to the discrete-time ELM:

$$
G\left(z^{-1}\right)=\frac{-0.0021 z^{-1}+0.0010 z^{-2}}{1-1.5851 z^{-1}+0.6197 z^{-2}}
$$

which was used for computation of the predictive controller in Equation (25). Zero mean white noise $e(t)$ is not take into account.

\section{SIMULATION RESULTS}

Both control strategies were verified by simulation experiments. The sampling period was set to $T_{v}=0.3 \mathrm{~min}$, all simulations took $500 \mathrm{~min}$ and 5 different step changes $w=[2,-1,1-1,1.5]$ were done during this interval.

The first simulation study was done for adaptive controller with the various values of the root $\alpha_{i}=0.05,0.1$ and 0.2 in Equation (32). The predictive controller was verified again for different values of the weighting factor $\lambda_{u}=0.05,0.5$ and 2 in (16).

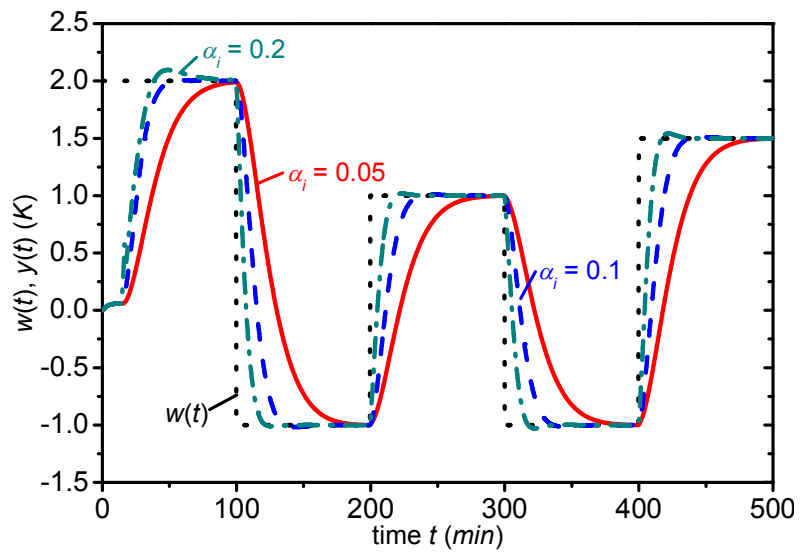

Fig. 3: Course of the reference signal $w(t)$ and the output variable $y(t)$ for various of the root $\alpha_{i}$ in adaptive control

Figures 3 and 4 represents simulation results for adaptive control. It can be clearly seen, that the increasing value of the parameter $\alpha_{i}$ results in the quicker output response, $y(t)$, but small overshoots. On the other hand, lower value of this root position is parsimonious to the input variable, $u(t)$, in Fig. 4 which could be in this case considered as a twist of the valve on the feeding of the cooling pipe.

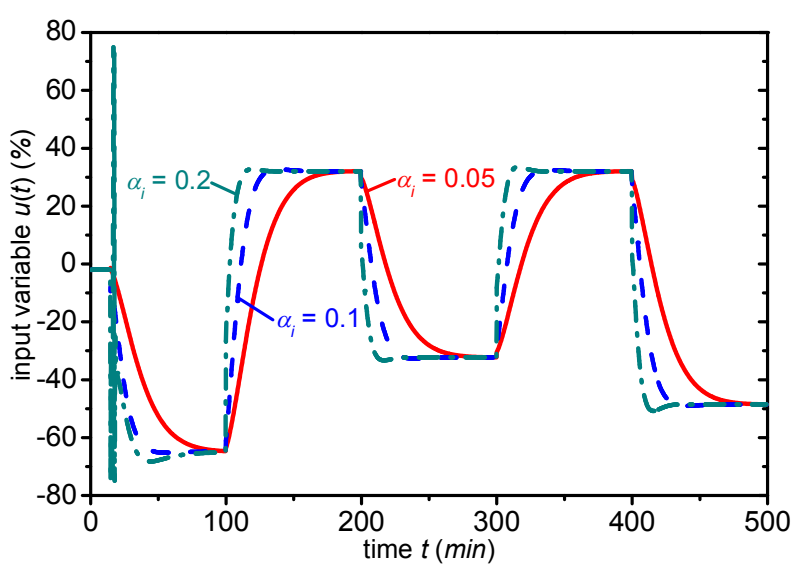

Fig. 4: Course of the input variable $u(t)$ for various of the root $\alpha_{i}$ in adaptive control

The quality of control was evaluated by the quality criteria $S_{u}$ and $S_{y}$ computed for a time interval as:

$S_{u}=\sum_{i=2}^{N}(u(i)-u(i-1))^{2}[-]$

$S_{y}=\sum_{i=1}^{N}(w(i)-y(i))^{2}\left[K^{2}\right] \quad$, for $N=\frac{T_{f}}{T_{v}}$.

The results for both control strategies are shown in Table 2.

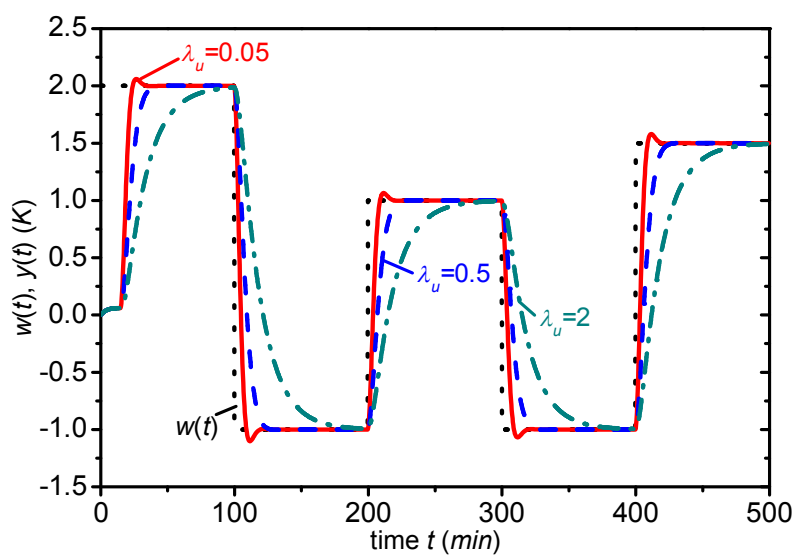

Fig. 5: Course of the reference signal $w(t)$ and the output variable $y(t)$ for various of the root $\lambda_{u}$ in predictive control

The simulation results of the second control strategy, predictive control, are shown in Fig. 5 and Fig. 6. This controller is tuned via weighting parameter $\lambda_{u}$ which is constant during the control and the second weighting parameter is $\delta_{u}=1$ as it is written above in the theoretical part. In this case, increasing value of the $\lambda_{u}$ results in slower response of the output variable $y(t)$.

The last two graphs in Fig. 7 and Fig. 8 compares the best results for both control strategies - adaptive control with $\alpha_{i}=0.1$ and predictive control with $\lambda_{u}=0.5$. We can say, that in this case both control strategies are comparable but the output response $y(t)$ in the predictive control reaches the reference signal (wanted value) $w(t)$ a little bit quicker than the adaptive controller. 


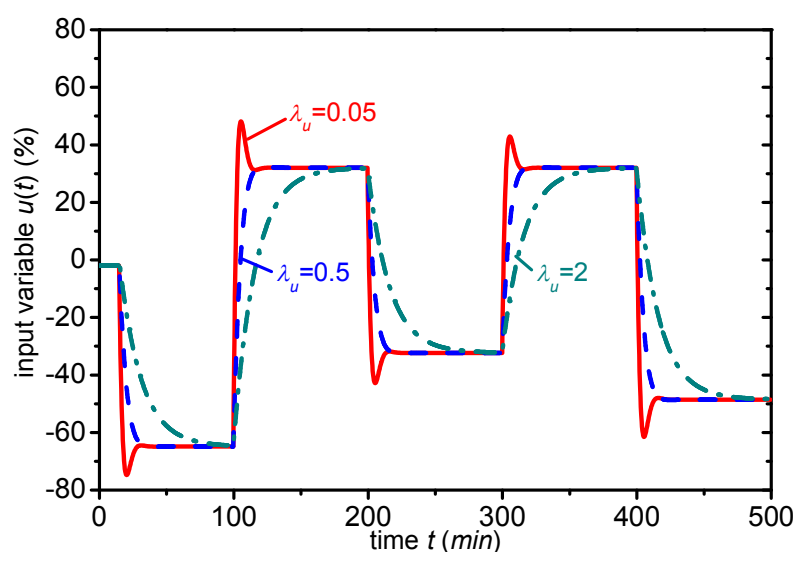

Fig. 6: Course of the input variable $u(t)$ for various of the root $\lambda_{u}$ in predictive control

Table 2 The results of control quality criteria $S_{u}$ and $S_{y}$

\begin{tabular}{|c|c|c|c|c|c|c|}
\cline { 2 - 7 } \multicolumn{1}{c|}{} & \multicolumn{3}{c|}{ Adaptive control, $\boldsymbol{\alpha}_{\boldsymbol{i}}=$} & \multicolumn{3}{c|}{ Predictive control, $\boldsymbol{\lambda}_{\boldsymbol{u}}=$} \\
& 0.05 & 0.1 & \multicolumn{1}{c|}{0.4} & 0.05 & \multicolumn{1}{c|}{0.5} & \multicolumn{1}{c|}{2} \\
\hline$S_{u}[-]$ & 192.4 & 492.3 & 8571.5 & 3265.1 & 868.3 & 265.3 \\
\hline$S_{y}\left[K^{2}\right]$ & 1664.4 & 934.7 & 532.7 & 430.3 & 653.3 & 1194.8 \\
\hline
\end{tabular}

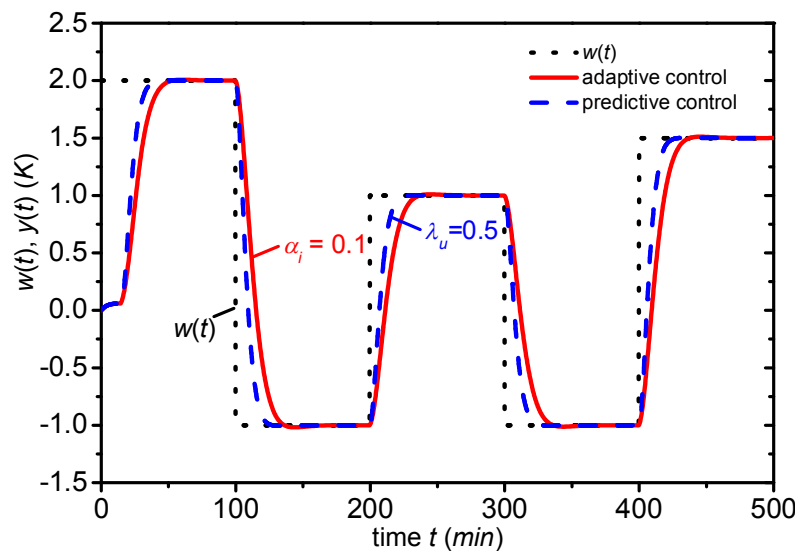

Fig. 7: The best courses of the output variable $y(t)$ for adaptive and predictive control

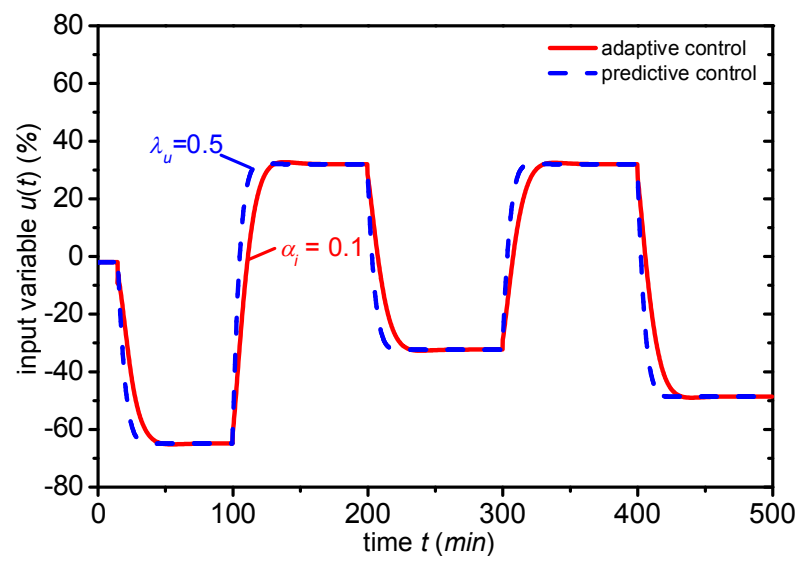

Fig. 8: The best courses of the input variable $u(t)$ for adaptive and predictive control

\section{CONCLUSIONS}

This paper presents two approaches which could be used for controlling of the temperature of the reactant inside the CSTR which is typical member of the nonlinear process with lumped parameters. Both, adaptive and predictive, controllers have good control results although the system has negative properties from the control point of view. The adaptive controller could be tuned by the parameter $\alpha_{i}$ while predictive controller has its weighting factor $\lambda_{u}$ as a tuning parameter too. Final comparison of both control techniques results better for the predictive controller but the difference is minimal. The future work will be focused on the verification of the obtained results on the real plant which could increase reliability of these methods.

\section{ACKNOWLEDGMENT}

This work was supported by the Ministry of Education of the Czech Republic under grants No. MSM 7088352101 and No. $1 \mathrm{M} 0567$

\section{REFERENCES}

Bobál, V., Böhm, J., Fessl, J., Macháček, J. (2005) Digital Self-tuning Controllers. Algorithms, Implementation and Applications. Springer

Corriou, J.-P. (2004). Process control. Theory and applications. Springer-Verlag London.

Clarke, D.W., Mohtadi, C., Tuffs, P.S. (1987) Generalized predictive control - Part I. The basic algorithm. Automatica. 23(2), 137-148

Chen, H., Kremling, A., Allgöwer, F. (1995) Nonlinear Predictive Control of a Benchmark CSTR. In: Proceedings of 3rd European Control Conference. Rome, Italy

Fikar, M., Mikleš J. (1999) System identification (in Slovak). STU Bratislava.

Kučera, V. (1991) Analysis and Design of Discrete Linear Control Systems. Prentice-Hall, London

Kučera, V. (1993) Diophantine equations in control - A survey. Automatica, 29, 1361-1375

Kulhavý, R., Kárný, M. (1984) Tracking of slowly varying parameters by directional forgetting, In: Proc. 9th IFAC World Congress, vol. X, Budapest, p. 78-83

Middleton, R.H., Goodwin, G.C. (2004) Digital Control and Estimation - A Unified Approach. Prentice Hall, Englewood Cliffs.

Mukhopadhyay, S., Patra, A.G., Rao, G.P. (1992) New class of discrete-time models for continuos-time systeme. International Journal of Control, vol.55, 1161-1187

Stericker, D.L., Sinha, N.K. (1993) Identification of continuous-time systems from samples of input-output data using the $\delta$-operator. Control-Theory and Advanced Technology, vol. 9, 113-125

Vojtesek, J., Dostal, P., Haber, R. (2004). Simulation and Control of a Continuous Stirred Tank Reactor. In: Proc. of Sixth Portuguese Conference on Automatic Control CONTROLO 2004. Faro. Portugal, p. 315-32 